\title{
Sprache im Alltag als Konstruktion von Lexikografie und Sprachwissenschaft
}

\author{
1 Fragen \\ 2 Vorklärungen \\ 3 Wissenschaftsgeschichtliche \\ Skizze \\ 4 Schlussfolgerungen \\ 5 Literatur
}

\section{Fragen}

In der Zeit vor der Institutionalisierung der Sprachwissenschaft in den Universitäten bestand sprachwissenschaftliches Handeln zu großen Teilen im Verfassen von Grammatiken, Rhetoriken, Wörterbüchern und sprachphilosophischen Arbeiten sowie in der induktiv entwickelten Texthermeneutik von Theologie, Rechtswissenschaft und Klassischer Philologie. Im Laufe des 19. und 20. Jahrhunderts diversifizierten sich die sprachwissenschaftlichen Tätigkeiten; zu den genannten traten weitere, zunehmend auch rein theoriebezogene Untersuchungen. Gegenstand aller dieser wissenschaftlichen Handlungsformen war und ist Sprache als System und Sprache als Gebrauch, wobei die Beschäftigung mit Sprache als Gebrauch von Systemeigenschaften nicht absehen kann, wohingegen sprachsystembezogene Arbeiten Bedingungen des Gebrauchs oft als etwas Externes ausschließen.

Thema meines Beitrags ist die diachron angelegte Frage nach denjenigen Gegenständen der Sprachwissenschaft, in welchen Spuren jener „Sprache-imAlltag" enthalten sind, die Herausgeberinnen und Herausgeber dieses Bandes soziologisch in Opposition zu Wissenschaft, Religion und Kunst definiert haben. Als exemplarischen Bereich sprachwissenschaftlichen Handelns wähle ich die Lexikografie, die eine der ältesten und an Textmuster gebundenen Formen der Sprachreflexion darstellt. Lexikografiegeschichte ist deshalb auch geeignet, viele der kaum reflektierten Vorannahmen der institutionalisierten Sprachwissenschaft der vergangenen zwei Jahrhunderte zu klären. Die Lexikografie ist zugleich ununterbrochen weiterentwickelt worden und nimmt auch in herausgehobener Weise an der jüngsten medialen Revolution teil. Die Anwendung der Fragestellung auf einige wenige Wörterbücher in Vergan- 
genheit und Gegenwart kann hier aber nur exemplarisch sein und soll in erster Linie einige methodologische Probleme bewusst machen.

\section{Vorklärungen}

Es ist nicht unbedingt damit zu rechnen, dass Sprache-im-Alltag in Wörterbüchern bzw. lexikografischen Programmschriften explizit thematisiert wird, und wenn, dann mittels anderer Bezeichnungen, die kaum vorherzusagen sind. Einen wichtigen Anhaltspunkt liefert die Wörterbuchbasis. Die schriftlichen Quellen werden aber erst mit zunehmender Verwissenschaftlichung der Lexikografe überhaupt benannt und sind vorher höchstens aus textpragmatischen Faktoren zu erschließen. Welche Textklassen erfüllen überhaupt die Bedingung, nicht wissenschaftliche, nicht künstlerische und nicht religiöse Sprache zu sein?

Die Wörterbuchbasis prägt nicht nur die äußere Selektion, sondern vor allem die Belegbeispiele. Ausführlichere semantisch-pragmatische Angaben sind eine relativ späte methodische Errungenschaft und daher weit weniger signifikant. Kompetenzbeispiele hingegen entziehen sich in der Regel dem durch die Wörterbuchbasis beschriebenen Parole-Ausschnitt. Auf der anderen Seite sind sie eine Informationsart, die auch außerhalb von Wörterbüchern in sprachwissenschaftlichen Texten vorkommt, sogar und mit Vorliebe in Theorietexten der Wittgenstein- wie der Chomsky-Tradition. Den Kompetenzbeispielen zu Lemmata bestimmter Wortschatzbereiche (s.u.) soll deshalb besondere Beachtung gelten.

Nach der von Herausgeberinnen und Herausgebern vorgenommenen soziologischen Bestimmung ist „Alltag“ nur dem Bereich der Wirtschaft benachbart und den Bereichen von Wissenschaft, Kunst und Religion entgegengesetzt. Bezogen auf den beschriebenen Wortschatz, auf Wörterbuchbasis und Kompetenzbeispiele ist diese Bestimmung einerseits zu weit und andererseits zu eng. Wären kaufmännische Korrespondenz und kaufmännische Briefsteller alltagssprachliche Wörterbuchquellen und Ausdrücke wie Aktie, Brutto und Wechsel alltäglich gebrauchte Lexeme? Wohl kaum; zurecht nannte man sie schon vor Leibniz Kunstausdrücke. Intentional gebildete oder semantisch fixierte und insofern „,künstliche“ Bezeichnungen gehören sicher nicht zu Sprache-im-Alltag (vgl. Kokemohr 1994, $231 \mathrm{ff}$.).

Nicht-Wissenschaft, Nicht-Kunst und Nicht-Religion sind aber auch: Rechtspflege und Verwaltung, die Politik, das Militär, die Seefahrt, die Jagd, der Gartenbau, der Sport, das Reisen u.a.m. Selbstverständlich findet in diesen Bereichen Alltagskommunikation statt, aber nicht ausschließlich. Wo der Sprachgebrauch institutionellen Bedingungen folgt, hört „Alltag“ auf: vor Gericht, im Parlament, beim Befehlen und überall dort, wo der Ausdruck von Gruppenzugehörigkeit notwendig ist. Auf der anderen Seite gehören alltägli- 
che Gesprächssituationen und Sprachhandlungsweisen ebenso $\mathrm{zu}$ wissenschaftlichen, religiösen und künstlerischen Institutionen und sind hier nicht einmal dominanter als dort, insofern der Sprachgebrauch in diesen Institutionen nicht durchweg reglementiert ist: Im Vatikan wird auch getratscht, in Fakuläten (unwissenschaftlich) gestritten, im Orchestergraben gelacht und in Galerien wie auf Buchmessen gefeilscht. Auch intentionaler, reflektierter und semantisch fixierter Sprachgebrauch ist außerhalb von Wissenschaft, Kunst und Religion keineswegs die Ausnahme: Der Skipper einer Segelyacht kann autoritativ festsetzen, dass „Mann über Bord!“ auch auf Frauen bezogen wird - Alltag im Sport.

Der oppositive Charakter der Definienten (Wissenschaft, Kunst, Religion) in der vorgegebenen Alltagsdefinition gilt offenbar nur für die institutionell normierten Gesprächssituationen und vor allem für die institutionell normierten Textsorten in Wissenschaft, Kunst und Religion - Disput, Abhandlung, Gebet -, und auch nicht für alle: Z.B. standen die Predigten vor allem der Reformationszeit dem Alltag näher als der Religion. Prüfungsgespräche oder das Verfassen von Wortartikeln sind wissenschaftlicher Alltag. Werbung ist für die Rezipienten Alltag, für die kreativen Macher aber wohl auch Kunst.

Am Erfolg versprechendsten scheint die Annäherung an Sprache-im-Alltag über typische Gesprächssituationen und deren lexikalische Elemente. Welche Gesprächstypen hierbei in Frage kommen, lässt sich mithilfe historiografischer Arbeiten wie Ariès/Duby (1989-1993) über das ,private Leben“ oder auch Kuczynski (1980) über den Alltag „des Volkes“ feststellen. Zwar ist nicht aller Alltag privat, aber fast alles Private ist alltäglich, insofern es routinierte Praxis ist (Kokemohr 1994, 231; Radtke 1994, 430). Alltag weitgehend ökonomisch zu begreifen und mit Wirtschaft gleichzusetzen, geht entweder von „Volk“ in Opposition zur ,herrschenden Klasse“ aus wie im historischmaterialistischen Ansatz Kuczynskis; ein Alltag der Herrschenden existiert in dieser Sicht nicht. Oder man setzt Alltag als etwas an, das stärker als Wissenschaft, Kunst und Religion ökonomisch bestimmt ist. Dies wäre aber zumindest für die Neuzeit zu bezweifeln, in der die Ökonomisierung nicht nur Universität, Kulturindustrie und Kirchen, sondern selbst den privaten und zwischenmenschlichen Bereich einzuholen droht.

Wenn Wörterbücher Sprache-im-Alltag berücksichtigen wollten, müssten sie bei den Quellen oder bei den angenommenen Benutzungssituationen Bezüge erkennen lassen zu konkreten Alltagssituationen: zu Gesprächen auf der Straße, auf dem städtischen Platz und unter der Dorflinde, auf dem Markt und beim Einkaufen, im Café und im Wirtshaus, in Vereinen und Clubs, in der Familie, in der Nachbarschaft, mit und unter den Dienstboten, in der Küche. Hier wird überall gegessen, getrunken und gespielt, gescherzt, gestritten und getratscht, geschimpft und befohlen. Der grooming talk, das Reden auf der Beziehungsebene, und die Imagearbeit spielen bei all diesen Situationen eine wichtige Rolle, mit Folgen für die vorzugsweise gewählten Themen. Sprache- 
im-Alltag heißt oft, übers Wetter reden, über das eigene (gesundheitliche) Ergehen und über andere Leute; erst danach über die anstehenden „Geschäfte“, verstanden als das, was man zu tun gedenkt oder was man gerade getan hat, also etwas, das nicht im engeren Sinne ökonomisch zu verstehen ist. Über Verallgemeinerungen kommt man von hier aus sehr schnell zu Weltdeutungen und Alltagstheorien als den dominanten Inhalten alltäglicher Kommunikationen (ein Beispiel interpretiert Kokemohr 1994, insbes. 236). Eine weitere, zentrale Funktion von Sprache-im-Alltag ließe sich geradezu als Verbreitung und Festigung alltagsweltlicher Theorien bestimmen.

Eine Definition von Sprache-im-Alltag, die der wissenschafts- und lexikografiegeschichtlichen Zielsetzung entgegenkommt, orientiert sich außer (1.) an den genannten Situationstypen (2.) an Sprachfunktionen, die der Herstellung und Wahrung sozialer Kontakte dienen. Solche Funktionen sind vorwiegend an bestimmte, nämlich dialogisch dominierte Situationstypen (im „Zeigfeld“) geknüpft. Kontaktfunktion und Situationstypen sind wiederum mit einigen Wortschatzbereichen besonders korreliert, ohne dass andere sich definitiv ausschließen ließen. Stellt man sich prototypisch den Smalltalk unter Nachbarinnen vor, sind einige Lexemklassen offensichtlich als funktional besonders wichtig zu identifizieren (deren terminologische Problematik hier ausgeklammert bleiben muss): Gesprächswörter (vgl. Radtke 1994, 424), Interjektionen, einige Modalpartikeln und weitere Ausdrücke der Modalität (Meyer 1994, 349), Phraseologismen bzw. Idiomatismen, Routineformeln, Sprichwörter, Heckenausdrücke, Gefühlswörter, Schimpfwörter, Verba sentiendi und emotive Verben; mundartlicher Wortschatz, je nach sozialer Stellung Argot/Slang und Grundwortschatz. Lexikografiehistorisch bietet sich nun folgendes Verfahren an: Man suche nach dialogisch formulierten Kompetenzbeispielen, die in alltägliche Gesprächssituationen wie die o.g. passen, beginnend mit der Suche in Wortartikeln zu Lemmata der zuletzt genannten Lexemklassen.

Die eingangs gestellten Fragen sind nun wie folgt zu präzisieren: Wie groß war/ist die Distanz der die jeweilige Wörterbuchbasis bildenden Kommunikationsformen und Varietäten zu vorstellbaren prototypischen Alltagssituationen? Beispielsweise erfüllen Zeitungstexte und Beschriftungen an und in Gebäuden und z.B. Verpackungen (Wandruszka 1994; Wienold 1994) in gewisser Weise die Kontaktfunktion und sind somit alltagsnäher als andere. Auf welche Situationen wird in dialogisch formulierten Kompetenzbeispielen bezug genommen? Mit diesen Vorklärungen soll nun ein diachroner Spaziergang durch Lexikografie und Sprachwissenschaft begonnen werden. 


\section{Wissenschaftsgeschichtliche Skizze}

Die Wörterbücher vom 8. bis zum 16. Jahrhundert sind von der allmählichen Emanzipation der „Volkssprachen“" vom Latein geprägt. Dies brachte ungewollt Spurenelemente alltagsnaher Textsortenstile in einige, aber nicht in alle Wörterbücher, die sich damit von der rhetorisch ausgefeilten antiken Literatur eines Cicero ein Stück weit entfernten. Auf den Abstand war schon Erasmus von Rotterdam peinlich bedacht. Seine ,Familiarum colloquiorum formulae“ (,Schülergespräche“) hatte er zunächst (1518) privat, für den Lateinunterricht seines Neffen verfasst. Als sie nachgedruckt und immer erfolgreicher wurden, gab er seine Verfasserschaft nur widerstrebend zu. Ein Ausschnitt in deutscher Übersetzung: „Wo kommst du her? - Aus dem Collegium Montaigu. Dann kommst du ja beladen mit Wissen einher. - Eher beladen mit Läusen.“ (Erasmus 1518/1982,13). Familiäre Gespräche schienen des Druckes nur würdig, wo es aus pädadogischen Gründen unumgänglich war. Die Opposition zur Literatur wurde, wie man sieht, aus der Sprachsituation des Mittellateins auf die des Deutschen übertragen und war nicht auf die Lexikografie beschränkt.

Schon vorreformatorisch zielten Wörterbücher wie der, Vocabularius praedicantium" von Johannes Melber (1477) teilweise auf die Produktion volkssprachlicher Predigten und vermehrten daher die volkssprachlichen Synonyme (Grubmüller1990, 2041). Dennoch blieb die Lexikografie des Humanismus auch in den deutschen Äquivalenten auf die lateinische Hochliteratur beschränkt, nur vereinzelt, und zwar in phraseologischen Syntagmen und satzwertigen Äußerungen wird die Realität des (deutschen) Alltagsdialogs sichtbar: „Wormit gadst vmb? was thůst?“ (Maaler 1561, 505v). „Meinst du das ich dir das yetz glaub/ daß sy vom Pamphilo schwanger gesein sey?“ (Maaler 1561, 183v).

Was Maaler erst vereinzelt nutzte, die Wörter in sprachrealistische und situativ zuordenbare Phrasen einzubetten und dabei die eigene Sprachkompetenz tätig werden zu lassen, wurde bei Hulsius' ,Dictionarium TeutschFranzösisch“ (1. Aufl. 1596, 3. Aufl. 1607) zur Notwendigkeit, weil es das erste Wörterbuch war, das Deutsch mit einer anderen europäischen Volkssprache parallelisierte (vgl. Wiegand 1998, 650). Die Notwendigkeit wurde bei Georg Henisch zum Prinzip: ,Teütsche Sprach und Weißheit" (1616) ist ein Wörterbuch der Gemeinplätze, das die Alltagstheorien am Vorabend des Dreißigjährigen Kriegs entfaltet. Allerdings sind hier mit Anspruch auf Allgemeingültigkeit formulierte, moralisierende Sentenzen (,Wenn ein Fürst jedermans gunst/ vnd das gemeine gebet verleurt/ so ists mit jhm geschehen." Henisch 1616, 1531) sehr viel häufiger als Äußerungen aus Dialogsituationen (,Was soll ich sagen“; „Ich bins nicht gewohnt“; „Solches geziemet dir gar nicht"; ebd. 1548; 1607; 1612). Phraseologismen als „gehobene“ Mündlichkeit? 
Ein Werk, das zu Unrecht nicht der lexikografischen Tradition zugerechnet wird, ist Comenius' ,Orbis pictus' (1659/1970), das für den Muttersprachunterricht von Kindern gedacht war und deshalb eine lexikografiehistorisch seltene Nähe zur Sprache-im-Alltag des Schulunterrichts aufweist. Der Rahmentext des ,Orbis pictus“ ist dialogisch (,Komm her, Knab, lerne klug sein! - Was ist das, klug sein?" Comenius 1659/1970, 69), der Hauptteil darstellend („Den Menschen haben wir besehen; itzt laßt uns fortschreiten zur Kost des Menschen und zu den Handwerkskünsten, welche hierzu dienen." Ebd. 125). Inwieweit die beschreibend erschlossenen Themen die Alltagswelt der Zeit abdecken, wäre eine eigene Untersuchung wert.

Mit den Wörterbüchern von Kaspar Stieler und Matthias Kramer wurde die normative barocke Sprachtheorie in Wortschatzdarstellung umgesetzt, deren schon personelle Nähe zur Dichtung unbestritten ist. Stielers Wörterbuch stellte sich geradezu in den Dienst einer dichterischen Sprach(system)entfaltung, während Kramer seine Arbeit weitgehend an den Bedürfnissen Handlungsreisender orientierte. Es überrascht daher, dass beide eine Fülle von Elementen alltagsweltlicher Dialoge vermitteln, die außerdem, bei Kramer eher als bei Stieler, mit den Alltagstheorien biblischer Phraseologismen angereichert sind:

O Gott! O Pferd ... O weh! wie ist mir so übel! (Stieler 1691 II, 1369). Halt die Schnauze! (ebd. II, 1906). Er hat mit ihm selbst zuschaffen (ebd. II, 1710). Das Mensch ist schön genug. (ebd. II, 1754). so gehts: schlab/ schlab! (ebd. II, 1800).

er sitzt besser zu Tisch als zu Pferde (Kramer 1700/1702, Bd. II, 1091a); du must deine Pfoten zuerst in der Schüssel haben (ebd. 209c). siehe da! der saubere Vogel (ebd. 739a). es glaubts kein Mensch (ebd. 47a). last ihn auspoltern/ bis er gnug hat/ (ebd. 223b). Mein Mann ist nicht zu Hause (ebd. 21a). Wie oft muß man dir ruffen? (ebd. 380a)

Steinbach (1734) bemühte sich offensichtlich um die Dokumentation des Sprachüblichen auch, aber nicht ausschließlich in der Literatur. Ein Bewusstsein für die soziale und situative Differenzierung sprachlicher Mittel war offenbar vorhanden. Die zeitgenössische und die spätere sprachhistorische Rezeption akzentuierte allerdings die Repräsentation der schlesischen Poeten in Steinbachs Wörterbuch stärker als die alltagsnahen Phraseologismen (Wiegand 1998, 658ff.). Kompetenzbeispielen mangelt die Autorität und Vorbildhaftigkeit, die Belegbeispiele schon aufgrund ihrer Schriftgebundenheit und daraus resultierender Nachweisbarkeit besitzen.

Mit Adelung (1793-1801) vollzieht sich ein folgenschwerer Wandel: Sprache-im-Alltag wird von ihm weitgehend mit ,bloß dem Volke eigenen Wörter(n) und Ausdrücke(n) (ebd. Bd. I, III), ,Wörtern und Formen des niedrigen Lebens“ (ebd. IV) gleichgesetzt und der ,,in Schriften üblich(en)“ „Hochdeutschen Mundart" (ebd.) entgegengesetzt. Die fünf „Schreib-“ und „Sprecharten“, darunter ,3. Sprechart des gemeinen Lebens und vertraulichen Umganges" (zit. ebd. $\mathrm{V}^{*}$ ), die Adelung schon in der 1. Auflage unterschied, sind 
mehr als bloße Stilebenen (Wiegand 1998, 674), nämlich der Versuch, Sprecher-Hörer-Konstellationen auf Ausdrucksebene abzubilden und dort zu klassifizieren - den Ausdruck Classen bezieht Adelung sowohl auf soziale Gruppen als auch auf die ebenfalls hierarchisch angeordneten "Schreibarten“. Aber dadurch macht Adelung es der Rezeption leicht, Sprache-im-Alltag als Stilkategorie ,Alltagssprache' zu begreifen und zu tradieren. Alltägliche Äußerungen („Aus was für einem Grunde glaubst du das?" Adelung 1793-1801, Bd. II, 828; „Was ich Ihnen sage, er ist wirklich todt.“; ebd. 1395) gehören für Adelung der „gemeinen“, „vertraulichen“ oder gar der „niedrigen Sprechart" an, die hier lexikografisch als Hintergrundfolie für die sozial wie regional idealisierte Hochsprache dient. Für die Veranschaulichung der Norm wird ein „So nicht!“, werden alltagsweltliche Äußerungen benötigt. Es sind dies bei Adelung aber bekanntlich nicht die ganz niedrigen und „pöbelhaften“, sondern solche der bildungsbürgerlichen Konversation: „Ich fand ihn unter den Büchern geschäftig“ (ebd. 603); „Sich auf die Geschichte legen“ (ebd. 606); „Was für Bücher liesest du?“ (ebd. 1393); „Die Gäste sind gekommen“ (ebd. 1696); „Wir haben einander erst neulich kennen gelernt“ (ebd. 1548); „Wie kommen Sie denn heute auf diesen Einfall?" (ebd. 1697).

Zum bildungsbürgerlichen Alltagsgespräch gehörte um 1800 auch Zeitungslektüre; sie ist bei Adelung zu typischen Kompetenzbeispielen geronnen: „Hollands Handel ist seit einiger Zeit gar sehr gefallen. Frankreich sucht dem Handel in seinen Staaten wieder aufzuhelfen." (ebd. 947). Zeitungen, so wird hier deutlich, werden nicht der Literatur im Sinne der Schriftsprachproduktion zugeordnet, sondern dem bürgerlichen Alltagsgespräch.

Die bis heute wirksame Hierarchisierung der Stilebenen wurde bei Adelung und auch bei Campe durch eine neue, gleichwohl elitär-hierarchische Ständegliederung unterstützt. In der Wahrnehmung der Sprachgemeinschaft wie der Sprachgeschichtsschreibung gerät infolgedessen Alltag dauerhaft in Opposition zu Literatur, zu Schriftlichkeit, zu Bildung und in Nachbarschaft zu allen unterbürgerlichen Schichten, zu Mündlichkeit und zum Mundartgebrauch. Dabei zeigen heutige Versuche, ,Literatursprache“ zeitunabhängig zu definieren (Radtke 1994, 424f.; Betten 1999), dass eine Sprache der Literatur kaum von anderen Existenzformen abzugrenzen ist. Ähnlich ist es mit der Trennung von Schriftlichkeit und Mündlichkeit. Die Entwicklung der Literalität in Deutschland zeigt (Knoop 1994, bes. 866ff.), dass die selbstverständlich scheinende Grenzlinie zu mäandern beginnt, je schärfer man hinsieht: Geschriebenes schließt Mündliches ein, u.U. sogar aus literarischem Gestaltungswillen heraus. Andererseits basiert vieles Mündliche, wie Gespräche über Bücher oder Zeitungsnachrichten, auf der Literalität einer Gesellschaft, nicht erst in Zeiten des Telefons. Die Oppositionen, die Sprache-imAlltag bestimmen sollen, sind lange schon konstruiert.

Mit der Etablierung einer standardsprachlichen Norm um 1800 begann auch die Mundartenlexikografie, die von vornherein auf nicht-schriftsprach- 
liche Quellen angewiesen war. Joh. Andreas Schmeller verarbeitet wohl erstmals systematisch gesammelte Äußerungen aus der Landbevölkerung und insbesondere von bayerischen Rekruten. Im Wörterbuch tauchen solche mehr oder weniger bearbeiteten Hörbelege der Sprache des ,gemeinen Mannes“ (so markiert in Schmeller/Frommann 1872/1985, Bd. I, 169) neben den - bedeutend zahlreicheren! - Literaturbelegen auf, etwa „Gê wèg, du irrst mi' dà!“ (Schmeller/Fromman 1872/1985, Bd. I, 131) oder „Das Kleid, die Schueh engen mich" (ebd. 106). Auch in der Mundartlexikografie wirkte offenbar die literatursprachliche Norm, ging es hier doch durchaus auch um den Nachweis der literarischen Qualität und literatursprachlichen Dignität der Mundarten.

Die frühen Lexikografen des Grimmschen Wörterbuchs standen dem ,derben“ und „kraftvollen“ Ausdruck bekanntlich positiv gegenüber, sofern er der nun romantisch verstandenen Poesie zuzuordnen war. Jacob Grimms PoesieBegriff umschloss auch rechts- und damit gebrauchssprachliche wie mundartliche Quellen (Weistümer, Urbare, Chroniken), sie mussten nur ,alt genug“ sein. Spätere Bearbeiter wandten sich einem an der Weimarer Klassik orientierten Literatursprachbegriff zu.

In den ersten Jahrzehnten des 19. Jahrhunderts etablierte sich die deutsche Philologie, und hatte dabei, wie jede neue Disziplin, ihre Existenz zunächst dadurch zu legitimieren, dass sie zum zentralen Forschungsgegenstand wählte, was einen allgemein anerkannten, natürlichen oder heiligen Wert repräsentierte. Was in der Geschichte der Chemie (Alchimie) Gold und Silber waren, war für die junge deutsche Philologie das Nibelungenlied; an ihm mussten sich weitere, der Forschung würdige Gegenstände messen lassen.

Beide Punkte sind Ursache dafür, dass Sprache-im-Alltag in den Bänden der ersten Bearbeitungsphase des ,Deutschen Wörterbuchs' nur selten, aber dennoch ihren Niederschlag fand. Die anfangs viel zu schmale Quellenbasis nötigte Jacob und Wilhelm Grimm ersatzweise zu illustrierenden Kompetenzsyntagmen, seltener -sätzen, wie s.v. abreise: „unsre abreise ist auf morgen festgesetzt" oder s.v. befallen „was befällt dich, dasz du so redest?“. Dass sich hierbei auch subjektive Spracherfahrungen bzw. -reminiszenzen Bahn brachen, zeigt eine Angabe wie s.v. Amtmännin: ,unsere sel. Mutter ... hiesz beim volk nur die framtmännin", die von Kritikern abgelehnt wurde.

Daniel Sanders, ganz im Geist von „1848“, negierte in seinem ,Wörterbuch der deutschen Sprache' (1859-1863) weitgehend die Existenz soziopragmatisch begründeter „Sprecharten“ zugunsten einer breiten, auch Zeitungen und anderes „Tagesschrifttum“ einschließenden Literatursprache (HaßZumkehr 1995, 220f.). Infolge dessen ist auch die „Sprache-im-öffentlichenAlltag" in seiner außerordentlich breit gestreuten Wörterbuchbasis enthalten; Belege aus Zeitungen werden hier erstmals ebenso durch Nachweis ,geadelt“" wie solche aus den Texten der „mustergültigen Schriftsteller“. Spuren gesprochener, dialogischer Sprache verblassen hingegen mehr und mehr; Mundartli- 
ches wird nahezu ausgeschlossen und Kompetenzbeispiele sind i.d.R. auf Syntagmen unterhalb der Satzgrenze beschränkt.

In der Sprachwissenschaft hatte Sprache-im-Alltag vom Ende des 19. Jahrhunderts bis nach 1945 generell keine Chance: Junggrammatik und Strukturalismus waren höchstens mit diachronen Zielen an einzelnen System-Aspekten der Mundarten interessiert; ansonsten genügten diejenigen Existenzformen von Sprache, die auch Gegenstand von Mediävistik und Literaturwissenschaft waren; dienten sie doch ,nur ${ }^{\star}$ zur Illustration von Theorien über das Sprachsystem und waren im Grunde austauschbar. In den germanistischen Schwesterdisziplinen musste in dieser Zeit zuerst die Emanzipation der jüngeren und Gegenwartsliteratur von der mit Goethe zuende gehenden Blütezeit der deutschen Literatur bewältigt werden. So blieb auch die Grammatikografie und Lexikografie eines Hermann Paul auf eine literarische Basis beschränkt.

Was Radtke $(1994,427)$ für die Romanistik feststellt, gilt mutatis mutandis auch für die Germanistik: Gebrauchstexte werden dort und nur solange als Ersatz für ,hohe' Literatur akzeptiert, wo letztere nicht überliefert ist. Ganze Subdisziplinen geraten in den Sog der Abwertung der Gebrauchsliteratur, wenn zu ihren Gegenständen aus irgendwelchen (historischen) Gründen kaum Hochliterarisches zählt. Dies betraf u.a. die niederdeutsche Philologie, die sich zu Beginn des 20. Jahrhunderts einen Platz im Fächerkanon zu erkämpfen begann.

1928 kündigte Agathe Lasch, Lexikografin und Grammatikografin des Mittelniederdeutschen, an der Hamburger Universität ein „Kolloqu[ium] über Fragen d[er] Alltagsspr.[ache]" an. Auch in anderen ihrer Publikationen äußerte sie sich am Rande über „Zweckliteratur“, d.h. zwar „längst beachtete, doch noch immer nicht sprachlich ausreichend gewürdigte [...] Texte" (Lasch 1932, 60, Anm. 3) rechts- und verwaltungssprachlicher Art, die für die Erforschung des Mittelniederdeutschen, der „Sprache der Hanse“, von herausragender Bedeutung waren. Laschs Vorsto $ß$ ist interessant, weil er die Folgen sichtbar macht, die zum Einen auf die fachübergreifende Fixierung auf Literatur- und geschriebene Standardsprache als überregionale und stilistisch gehobene Varietät, zum Zweiten auf die Indifferenz zurückzuführen ist, die eine systemorientierte Sprachforschung gegenüber den sprachlichen Existenzformen, die ihre empirische Fundierung liefern, an den Tag legt.

Eine Wende zeichnete sich ab, als die Ordinary Language Philosophy ihren Einfluss auch auf die Sprachwissenschaft auszuüben begann. Eine bestimmte Form der Sprache-im-Alltag erschien nun philosophisch geadelt und - zumindest innerhalb der germanistischen Linguistik - der Literatur- oder Standardsprache an Prestige und wissenschaftlichem Nutzwert gleichgestellt. Wittgenstein veranschaulicht in den ,Philosophischen Untersuchungen“ (I, Nr. 27)

1 Ich danke Frau Dr. B. Wägenbaur vom Deutschen Literaturarchiv in Marbach a. N. für den Zugang zu den Vorlesungsverzeichnissen. 
anhand von Ein-Wort-Sätzen, was man generell mit Sätzen tun kann: „Wasser! Fort! Au! Hilfe! Schön! Nicht!“. Diese alltäglichen Sprachhandlungen stehen nicht nur in Opposition zur Philosophie- und Wissenschaftssprache, deren Kritik sich die Ordinary Language Philosophy zum Ziel gesetzt hatte, sondern auch zur standardsprachlichen Norm, nach der schon Kinder lernen, dass man sich in ,ganzen Sätzen“ ausdrücken soll. Aber die Philosophie und die Linguistik in ihrem Gefolge weisen nun nach, dass es sich in einem neuen Verständnis um vollständige, d.h. ,gute“ Sätze handelt.

In der deutschen Fassung von Austins Vorlesungsnachschriften (,How to do things with words') werden nach wenigen Seiten Beispielsätze angekündigt, die ,ganz alltägliche Verben in der ersten Person ..." enthalten. Der erste von ihnen ist: ,Ja" ,als Äußerung im Laufe der standesamtlichen Trauung“ (Austin 1972, 26) und der vierte: „Ich wette einen Fünfziger, daß es morgen regnet" (ebd. 27). Während die erste Äußerung nach strikten institutionellen Regeln abläuft, dreht sich die zweite um typische britische Alltagsthemen. Natürlich spielen Varietäten oder Situationszugehörigkeit für Austin keine Rolle, denn die Beispiele werden allein um ihrer Performativität willen als typische Sätze der ,normalen“ Sprache untersucht.

Nicht nur die linguistische Pragmatik, auch das dieser entgegengesetzte Chomsky-Paradigma entdeckte Sprache-im-Alltag für die eigenen Zwecke. Das allererste Beispiel, das Chomsky in seinen,Aspects of the theory of Syntax ' (1965) bildet - die deutsche Ausgabe behält die englischen Beispielsätze bei -, gehört kontextuell, wie bei Adelung, in ein Gespräch über Bücher: „I called up the man who wrote the book that you told me about." (Comsky 1969, 22). Wo Bücher nicht mehr nur gelesen, sondern geschrieben werden, herrscht allerdings ein außergewöhnlicher Alltag: der von Intellektuellen. Die meisten Beispielsätze Chomskys und seiner breiten Nachfolgerschaft spiegeln neben dem Intellektuellen-Alltag die Alltagswelt von „John“ und „Bill“, die auffallend häufig mit Bier und Mädchen zu tun haben. Die Examinierung der Sprecherkompetenz zur Verifizierung der Generativen Theorie verlangt, so scheint es, eine Sprache des „vertraulichen Umgangs“ unter (männlichen) Linguisten: Hier kennt man sich so gut aus, dass man Grammatikalitätsgrade sicher differenzieren kann.

Verdankt denn, so könnte man fragen, Sprache-im-Alltag ihren Durchbruch als Gegenstand der Linguistik der unfreiwilligen Allianz aus linguistischer Pragmatik und Sprachsystemforschung in der Nachfolge Chomskys, die den Native Speaker, den Kumpel von John und Bill, als zuverlässigste Quelle sprachlichen Wissens inthronisierte? Befreite er die theoretische Sprachwissenschaft endlich von den Mühen der Textanalysen, empirischen Erhebungen und Auswertungen? Das Beste am Native Speaker ist, dass ihn jeder Linguist in sich selber finden und zum Diener beliebiger Zwecke machen kann. Zum Lohn widmete Coulmas dem Native Speaker 1981 eine eigene Festschrift 
(Antos 1996, 256ff.). Gottseidank hat der Native Speaker bis jetzt kein Wörterbuch geschrieben, wenn er wohl auch in einigen seine Finger drin hat.

Die linguistische Pragmatik diesseits der Sprachphilosophie hat den Reiz der individuellen, sozialen und situativen Vielfalt von Sprache-im-Alltag schnell entdeckt. Der allgemeine Bezugspunkt der Varietätenforschung hat sich seit den 70-er Jahren ganz sicher weg von der immer heterogener werdenden Literatursprache und hin zu einer mündlichkeitsnäheren Sprache-imöffentlichen-Alltag entwickelt. Gleichzeitig entwickelten sich in Linguistik wie Literaturwissenschaft Teildisziplinen, die sich der Sprache-im-Alltag widmeten: Gesprächsanalyse, oral history und mündliches Erzählen. Die Erforschung von Schrift und Schriftlichkeit scheint erst vor diesem Hintergrund Profil und interdisziplinäre Aufmerksamkeit zu gewinnen. Diese Entwicklung hatte auch Auswirkungen auf die zeitgenössische Lexikografie.

Während das ,Wörterbuch der deutschen Gegenwartssprache' durch seine Quellenbasis der literatursprachlichen Norm vor allem in stilistischer Hinsicht mit einem differenzierten Ebenenmodell verbunden blieb, räumt das ,Große Wörterbuch der deutschen Sprache' aus dem Duden-Verlag (1. Auflage 1976ff.) der „Umgangssprache“ einen nie zuvor dagewesenen Raum ein. In der Wörterbuchbasis sind nicht nur Brecht und Mann, Böll und Grass, Freud und Habermas vertreten, sondern auch die ADAC-Motorwelt, Heftchenromane, Männermagazine, Gütermanns Näh-Lexikon und die Sitzungsprotokolle des Bundestags. Die Stilebenen und ihre Bewertung werden freilich nicht aufgegeben. Mit ,ugs.“ und ,salopp“ markierte Äußerungen - sie entstammen überwiegend der lexikografischen Individualkompetenz - werden stets in die unmarkierte normalstilistische Ebene übersetzt.

Der quantitative Vergleich diverser Markierungen, die der Sprache-imAlltag mal näher, mal entfernter stehen, ergibt für die CD-Rom des UniversalDuden

- ugs.; umgangsspr.

7990

- mdal. [mundartlich]

- journal.

- kaufm.

- gehoben

- dichter.

- literar.

6
43
418
7665
528
152

Das Verhältnis von alltagsnäherer und alltagsfernerer Sprache liegt somit bei $8457: 8345$, d.h. es ist fast $1: 1$.

In der ebenfalls auf CD-Rom verfügbaren 9. Auflage von Hermann Pauls ,Deutschem Wörterbuch', das sich dezidiert der Dokumentation der deutschen Literatursprache verschreibt, summieren sich die alltagsnäheren Markierungen (ugs., journal., mdal., kaufm.) auf 1872 und die alltagsferneren (dicht., geh., literar.) auf 629; ihr Verhältnis ist somit grob 3:1. Paradox? Nein, wohl eher der Beleg dafür, dass im neuen ,Paul" die „Latte“ der unmarkierten Stil- 
ebene „höher gelegt“ wurde als im Duden, sodass unterhalb weitaus mehr zu markieren ist als oberhalb; der Duden hingegen ist den ,goldenen Mittelweg“" gegangen.

Festgehalten werden muss aber, dass Sprache-im-Alltag, obwohl nun breiter repräsentiert, lexikografisch immer noch als Stilebene und damit nicht wirklich angemessen behandelt wird. Dies könnte erst durch eine Situationsund Textsortenmarkierung geändert werden, die die Stilebenenmarkierung ersetzt. Die vor allem von Wiegand vorangebrachte lexikografische Theorie hat die (Un-)Angemessenheit semantisch-pragmatischer Angaben immer wieder an alltagsweltlichen Dialogen überprüft - und dem „Botel“ so einen wenigstens für seine Heidelberger Schülerinnen und Schüler unvergesslichen Ehrenplatz in der Wissenschaftsgeschichte gesichert. Dem berechtigten Nutzerinteresse an Orientierung über Stilnormen der Lexemverwendung müsste man freilich anderweitig entgegenkommen, wenn Sprache-im-Alltag über Situations- und Textsorten- statt über Stilmarkierungen bestimmt werden soll. Die Entwicklung der Techniken der Sprachaufzeichnung und der Recherchierbarkeit solcherart gewonnener Daten war in den letzten Jahrzehnten rasant und hat neue Möglichkeiten zur Erforschung von Sprache-im-Alltag eröffnet. Nicht nur immer größere Korpora geschriebener, sondern auch gesprochener Sprache, wie sie im Institut für Deutsche Sprache in Mannheim aufgebaut werden, zeugen von der durchgesetzten Gleichberechtigung aller situativen, sozialen und regionalen Varietäten.

\section{Schlussfolgerungen}

Nach diesem notgedrungen sehr groben und sehr exemplarischen Durchgang durch die Geschichte der germanistischen Sprachwissenschaft bzw. Lexikografie lässt sich festhalten, dass die oppositive Bestimmung von Sprache-imAlltag seit dem Humanismus existiert, dass die Wissenschafts-, bzw. die mit ihr verknüpfte Sprach- und die Gesellschaftsgeschichte zur Verschärfung diverser, nämlich stilistischer wie varietätenbezogener Oppositionen wesentlich beigetragen hat.

Für eine historische Erforschung von Sprache-im-Alltag sind Wörterbücher mithin nur sehr eingeschränkt zu gebrauchen. Andererseits gibt es nicht viele andere Quellen, die sie diesbezüglich ersetzen könnten; hier kommen wesentlich die unpublizierten infrage. Überraschend ist allerdings, dass ausgerechnet die barocke Lexikografie sich als ein noch unentdeckter Fundus alltagsweltlicher Sprachformen erwiesen hat, von deren "Theoriengehalt" so manches die Zeiten überdauert hat:

die Welt ist rund/ wer nicht darinnen schwimmen kann/ der gehet zu Grund (Kramer Bd. II, 394c). 


\section{Literatur}

Adelung, Johann Christoph (1793-1801): Grammatisch-kritisches Wörterbuch der hochdeutschen Mundart, Leipzig. Nachdruck hg. und mit einer Einleitung vers. von Helmut Henne. Hildesheim 1975.

Antos, Gerd (1996): Laien-Linguistik. Studien zu Sprach- und Kommunikationsproblemen im Alltag. Am Beispiel von Sprachratgebern und Kommunikationstrainings. Tübingen.

Ariès, Philippe; Duby, George (1989-1993) (Hgg.): Geschichte des privaten Lebens. 5 Bde. Frankfurt (Orig.-ausg. Paris 1986).

Austin, John L. (1972): Zur Theorie der Sprechakte (How to do things with Words). Deutsche Bearbeitung von Eike von Savigny. Stuttgart.

Betten, Anne (1999): Sprachgeschichte und Literatursprache. In: Andreas Gardt/Ulrike Haß-Zumkehr/Thorsten Roelcke (Hrsg.): Sprachgeschichte als Kulturgeschichte. Berlin, 73-87.

Chomsky, Noam (1969): Aspekte der Syntax-Theorie. Aus d. Amerikan. übersetzt und hrsgg. von einem Kollektiv unter d. Leitung v. Ewald Lang. Berlin. Frankfurt a.M.

Comenius, Johann Amos (1659/1970): Orbis sensualium pictus. In: ders., Opera omnia Bd. 17, hrsgg. von Jaromír Červenska, Stanislav Králík, Jiři Kyrášek. Akademie der Wissenschaften, Prag.

Duden - Das Große Wörterbuch der deutschen Sprache (1976ff.). 6 Bde. Mannheim.

Duden - Deutsches Universalwörterbuch. Mannheim, 1. Aufl. 1983. [3. Aufl. auch als CD-Rom 1996/97].

Erasmus von Rotterdam (1518/1982): Familiarum colloquiorum formulae. Schülergespräche. Lateinisch/deutsch. Ausgewählt, übersetzt und herausgegeben von Lore Wirth-Poelchau. Stuttgart.

FS Stempel $(1994)=$ Sprachlicher Alltag. Linguistik - Rhetorik - Literaturwissenschaft. FS für Wolf-Dieter Stempel zum 7. Juli 1994, hrsg. von Annette Sabban und Christian Schmitt. Tübingen.

Grubmüller, Klaus (1990): Die deutsche Lexikographie von den Anfängen bis zum Beginn des 17. Jahrhunderts. In: Wörterbücher. Ein internationales Handbuch zur Lexikographie. 3 Teilbde., hg. v. Franz Josef Hausmann, Oskar Reichmann, Herbert Ernst Wiegand, Ladislav Zgusta. Berlin, 2. Teilbd., 2037-2049.

Haß-Zumkehr, Ulrike (1995): Daniel Sanders. Aufgeklärte Germanistik im 19. Jahrhundert. Berlin, New York.

Henisch, Georg (1616): Teütsche Sprach und Weißheit. Augsburg.

Knoop, Ulrich (1994): Entwicklung von Literalität und Alphabetisierung in Deutschland. In: Schrift und Schriftlichkeit. Ein interdisziplinäres Handbuch internationaler Forschung. Writing and its Use. Hrsg. von Hartmut Günther, Otto Ludwig, zusammen mit Jürgen Baurmann. Berlin, New York. Halbbd. 1, 859-872.

Kokemohr, Rainer (1994): „C'est la crise!“. Zur Funktionsweise alltagsrhetorischer Weltdeutungen. In: FS Stempel, 227-244.

Kramer, Matthias (1700/1702): Herrlich-großes Teutsch-Italiänisches Dictionarium, 2 Bde., 2. Auflage 1700 bis 1702 (1. Auflage 1678). Nachdr. mit e. Einf. hg. von Gerhard Ising. Hildesheim 1982.

Kuczynski, Jürgen (1980): Geschichte des Alltags des deutschen Volkes 1600-1945. Studien. 5 Bde. Köln (Lizenzausgabe nach Berlin 1980).

Lasch, Agathe (1932): Die altsächsischen Psalmenfragmente. In: Nd. Studien, FS C. Borchling, Neumünster, 229-272. Wiederabgedr. In: Timothy Sodmann (Hg.): Agathe Lasch. Ausgewählte Schriften zur niederdeutschen Philologie. Neumünster 1979. 
Lehr, Andrea (1999): Quo vadis, Sprachwissenschaft? Sprache - Sprachwissenschaft Öffentlichkeit: Randnotizen zur IDS-Jahrestagung 1998. In: ZGL 27, 38-66.

Lübbe, W./Rentsch, Th. (1984): Ordinary Language Philosophy. In: Historisches Wörterbuch der Philosophie, hrsg. von Joachim Ritter und Karlfried Gründer. Basel. Bd. 6, 1246-1247.

Maaler, Josua (1561): Die Teutsch Spraach. Nachdr. d. Ausg. Zürich 1561. Mit e. Einf. hg. v. Gilbert de Smet. Hildesheim 1971.

Meyer, Wolfgang J. (1994): Charles Bally über Modalität. In: FS Stempel, 439-364.

Paul, Hermann (1992): Deutsches Wörterbuch. 9., vollständig überarbeitete Aufl. von Helmut Henne und Georg Objartel unter Mitarbeit von Heidrun Kämper-Jensen. Tübingen 1992 (auch als CD-Rom).

Radtke, Edgar (1994): „Alltag“ in der Italienischen Sprachgeschichtsschreibung. In: FS Stempel, 419-433.

Schmeller, Johann Andreas und G. Karl Fromman (1872/1985): Bayerisches Wörterbuch. 2 Bde. In vier Teilen. Nachdr. der von Karl Frommann bearbeiteten 2. Ausg. München.

Stieler, Kaspar (Der Spate) (1691): Der Teutschen Sprache Stammbaum und Fortwachs oder Teutscher Sprachschatz/ [...]. Nürnberg 1691. Nachdr. hg. und mit e. Nachwort vers. von Stefan Sonderegger. München 1968.

Wandruszka, Ulrich (1994): Zur Semiotik der Schlagzeile: Der Kommunikationsakt „Meldung“. In: Festschrift Stempel, 571-589.

Wiegand, Herbert Ernst (1998): Historische Lexikographie. In: Sprachgeschichte. Ein Handbuch zur Geschichte der deutschen Sprache und ihrer Erforschung. 2., vollständig neubearb. und erw. Aufl. 1. Teilbd. (= Handbücher zur Sprach- und Kommunikationswissenschaft; 2.1), 643-715.

Wienold, Götz (1994): Inscriptions in daily life. In: Festschrift Stempel, 635-652.

Wittgenstein, Ludwig (1958/1975): Philosophische Untersuchungen. Frankfurt a M.

Wörterbuch der deutschen Gegenwartssprache (1961ff.), hgg. von Ruth Klappenbach und Wolfgang Steinitz. In 6 Bänden. Berlin. 